\author{
Kiss, Gergely PhD*
}

gpetit.gergely@gmail.com

associate professor, University of Pécs, Hungary

\title{
Les Fasti Ecclesiae Gallicanae
}

Présentation d’une entreprise prosopographique en évolution

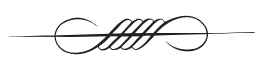

- Fasti Ecclesiae Gallicanae. Presentation of a Prosopographical Project in Progress -

Abstract The present paper aims to show briefly the formation of the research project Fasti Ecclesiae Gallicanae founded by Hélène Millet in 1990, their antecedents, the concept, the structure and the constitution of the volumes to have a general idea not only about the methods, the problems occurred, but the solutions applied as well. Finally, it takes into consideration its results and impacts in the world of medievalists.

Keywords auxiliary sciences, medieval prosopography, clergy, France thirteenth-fifteenth centuries, institutional history (Medieval Church)

DOI 10.14232/belv.2015.2.6 http://dx.doi.org/10.14232/belv.2015.2.6

Cikkre való hivatkozás / How to cite this article:

Kiss, Gergely (2015): Les Fasti Ecclesiae Gallicanae. Présentation d'une entreprise prosopographique en évolution. Belvedere Meridionale vol. 27. no. 2. 92-97. pp

ISSN 1419-0222 (print) ISSN 2064-5929 (online, pdf)

* L’auteur est membre du groupement de recherche MTA-DE „Lendület” Magyarország a Középkori Európában. Les recherches ont été supportées par le Fonds National Hongrois de la Recherche Scientifique - OTKA NN 109690. 


\section{Historique}

L'entreprise des Fasti Ecclesiae Gallicanae (FEG) est arrivée en 2014 à sa maturité pour plusieurs raisons. Quant aux préliminaires, on doit renvoyer aux premières impulsions du milieu des années 1970 où s'est accentuée l'importance de la prosopographie dans le travail des médiévistes. ${ }^{1}$ Le problème du stockage et du traitement informatisé des données s'est rapidement posé, de sorte que des tables rondes furent organisées au milieu des années 1980 sous l'égide du Centre national de la recherche scientifique (CNRS) pour aborder la question. ${ }^{2}$ Basé sur ces travaux préliminaires, le projet Fasti a été lancé par Hélène Millet en 1990. Le CNRS a entrepris le financement tandis que l'Institut de recherche et d'histoire des textes (IRHT) a accepté l'accueil du projet. Le programme des $F E G$, qui a débuté comme un groupement de recherche (GDR) autonome, a vécu depuis le milieu des années 1994 une reconfiguration institutionnelle sous forme de différents regroupements des équipes de recherche (1994-2001 : GDR Gerson, 20022009 : GDR Salvé ${ }^{3}$ ). Depuis 2010 il fonctionne comme " comité Fasti », c'est-à-dire une organisation fédérative où les différentes tâches se répartissent entre le responsable des Fasti au sein du LAMOP, ${ }^{4}$ l'administrateur de la base de données, le responsable de l'information et de la documentation et celui en charge de l'édition des volumes ${ }^{5}$.

\footnotetext{
1 Sans parler ici des premières ébauches comme la Gallia christiana (De SAINTE-MARHTE - HaurÉAU 1715-1865), Gallia christiana novissima (AlBANÈS - Chevalier 1899-1920) ou Duchesne 1907-1910. Cf. : Feg, Historique.

2 Millet 1985 ; Autrand 1986; cf. l'article d'Herminia VILAR dans le présent volume, surtout note nr. 11.

3 Cf.: Feg, Historique.

4 LAMOP est un acronyme : Laboratoire de Médiévistique Occidentale de Paris. Cf. : LAmop.

5 Cf. : Feg, Organigramme. La méthode de travail est toujours coopérative, les membres de l'équipe se réunissent deux fois l'an pour, outre les questions administratives, établir le bilan, présenter les travaux achevés et en cours. Cf. FEG, RÉUNIONS.
}

\section{Concept, structure, présentation des volumes}

Le projet FEG propose, tel que son promoteur l'a expliqué maintes fois et que rappelle le sous-titre que porte chacun des volumes, de répertorier les 130 diocèses (136 avec la Corse) de la France, correspondant à la structure territoriale établie au début du XIv ${ }^{\mathrm{e}}$ siècle. $^{6}$ Quant à la chronologie, elle repose d'une part sur les contraintes imposées par les sources même, qui deviennent plus informatives tout en faisant référence à des noms, depuis le début du XIII ${ }^{\mathrm{e}}$ siècle. D'autre part la fin du $\mathrm{Xv}^{\mathrm{e}}$ siècle est tenue traditionnellement comme la limite chronologique des époques médiévale et moderne.?

Le projet veut-il présenter seulement des listes d'évêques, de dignitaires et de chanoines ou ne cherche-t-il pas aussi à renouveler la notion de "fastes » ? La réponse est bien simple, les Fasti Ecclesiae Gallicanae sont une entreprise qui essaie toujours de situer les individus dans l'espace, dans le temps et dans le contexte local qui était leur diocèse. Plus les volumes paraissent, plus la mise en contexte spatiale et temporelle s'élargit.

André Vauchez avait raison de remarquer dans l'avant-propos du premier volume : «J'y vois un signe parmi d'autres d'un renouveau du goût pour l'érudition, sensible chez les médiévistes français depuis une quinzaine d'années. Précisons toutefois, pour éviter tout malentendu, qu'il s'agit bien d'une nouvelle érudition, qui exploite au mieux les immenses possibilités techniques offertes par l'informatique et trouve son inspiration dans une véritable problématique historique : celle de la prosopographie appliquée ici à la « micro-société

6 Millet 1996. IX.

7 Présentation du projet Fasti ecclesiae gallicanae de Jean-Michel Matz tenue à Szeged, le 17 septembre 2007. Je voudrais présenter ma gratitude à l'auteur de m'avoir procuré le texte. 
- mais si importante sur le plan qualitatif! - des dignitaires et des notables ecclésiastiques $»{ }^{8}$

"Informatique " et "prosopographie appliquée ", voici les deux mots-clés fondamentaux du projet auxquels on doit sans doute ajouter la notion de «micro-société ». Tout en profitant des avantages de l'informatique, le concept a prévu la création d'une base de données prosopographique avant tout pour stocker les informations portant sur les individus. Du fait de la mobilité des clercs médiévaux, cette structure aurait eu pour résultat une mise en parallèle des informations reprises diocèse par diocèse. Cependant la double structure des bases permet d'éviter ce problème. Les individus - points de départ référentiels - sont enregistrés dans la base du diocèse en question sous un numéro d'identification. Ce même individu peut être, bien entendu, retrouvé dans la base d'un autre diocèse sous un autre numéro d'identification. Les différentes entrées sont enregistrées dans une autre base cumulative qui permet de coiffer les informations fournies par les différentes bases diocésaines. ${ }^{9}$

Quant à la réalisation, les collaborateurs du projet préparant des notices biographiques collectent les informations selon un schéma prévu, constitué des éléments suivants : naissance et mort, ordres sacrés, carrière bénéficiale, études, autres renseignements, hypothèses d'identification, références. La structure uniforme permet d'avoir une présentation identique pour chacun des individus. Toutefois les volumes confectionnent bien plus que de simples listes des ecclésiastiques.

S'intéressant au diocèse entier, il présente avant tout la description des cadres institutionnels : la formation du diocèse, le chapitre cathédral et les collégiales, les doyennés, les

8 VAUChEZ 1996. VII.

9 Feg, Conception. En détail cf. : Millet - Raux 2007. Pour citer un exemple, on trouve Amanevus de Fargis qui est répertorié dans trois diocèses français (Amiens, Reims, Agen), dans les volumes 1, 3, et 5 sous différents numéros d'identification (Amiens : 0410, Reims : 1526, Agen : 197). archidiaconés et archiprêtrés. Cette notice institutionnelle est suivie par une autre partie, réservée au siège épiscopal et aux droits seigneuriaux de l'évêque puis aux officialités et au chapitre cathédral, permettant ainsi de s'enfoncer dans le cour de la société cléricale du diocèse puisque les dignitaires, les chanoines et les autres desservants complètent cet aperçu social. Il faut souligner que pour éviter toute confusion entre diocèses, les particularités sont précisées de façon exhaustive. Il est évident aujourd'hui que les diocèses, les chapitres, en bref les cadres institutionnels diffèrent les uns des autres, que ce soit l'espace même (configuration du palais épiscopal, du quartier canonial) la structure et la composition des dignitaires, la répartition des réguliers et des séculiers, le nombre des prébendes, etc. La présentation des sources propres et extérieures des diocèses est également indispensable pour offrir un panorama de la culture intellectuelle locale. Une bibliographie exhaustive termine la notice institutionnelle qui est donc très appréciable.

Elle est suivie des tables chronologiques qui font voir les évêques, leurs auxiliaires, les dignitaires du chapitre (en fonction de leur structure variée) et éventuellement les auxiliaires du chapitre en ordre chronologique suivi par le numéro d'identification des individus.

Les notices biographiques forment l'essentiel des volumes. Les notices des évêques offrent une série de biographies sous forme d'un texte intégral, mais structuré par des rubriques définis. ${ }^{10}$ Les notices réservées aux dignitaires, chanoines, prébendés et aux auxiliaires de l'archevêque ou de l'évêque sont introduites par l'explication des règles et abréviations utilisées pour la composition des notices issues

10 1) état civil, 2) formation, 3) carrière ecclésiastique, 4) accession au siège, 5) action pastorale, 6) carrière civile durant l'épiscopat, 7) transfert éventuel, 8) vie culturelle, religieuse, 9) décès, sépulture, 10) représentations contemporaines, 11) devise, armoiries, sceaux. 
de la base de données. Elle donne des renseignements sur la composition de la base, l'identification des individus, la dénomination et le classement des individus, la présentation des notices, les abréviations chronologiques, la liste des abréviations contenues dans les références, les codes diocésains en y ajoutant les particularités de chacun des volumes. Les notices contiennent les cas incertains et, le cas échéant, les clercs exclus, tous présentés dans des chapitres séparés.

Les indices offrent différents modes de recherche, soit par le nom des individus entrés dans la base, soit celui des diocèses concernés, ou le tableau des numéros d'identification, l'index des cas incertains et exclus de la base.

\section{Bilan, impact des FEG}

Bien qu'aujourd'hui le projet des Fasti Ecclesiae Gallicanae soit loin de couvrir l'intégralité des diocèses (dont seulement $10 \%$ sont publiés), il a déjà donné beaucoup d'impulsions aux autres entreprises en Europe, en particulier au Portugal et en Hongrie. ${ }^{11}$ Quant à la progression de l'entreprise en France, voici le tableau actualisé des volumes parus.

11 Quant au Portugal on renvoie ici à l'article d'Herminia VILAR dans le présent volume. En Hongrie, la monographie commémorant le millénaire del'évêché de Pécs a beaucoup inspiré des méthodes appliquées par l'équipe des Fasti. Fedeles - SARBAK - SüMEgi 2009. En outre, des tentatives de lancer un projet prosopographique sous forme d'un groupement de recherche ont été formulées depuis le milieu des années 2000 sans avoir un soutien financier. Pour ce qui est des résultats "individuels ", cf. KIss 2014., surtout p. 130-133. ; C. Tóth - Lakatos - Mikó 2014.

TABle 1 Les volumes publiés des Fasti ecclesiae Gallicanae ${ }^{12}$

\begin{tabular}{|c|c|c|}
\hline Vol. & Diocèse & Références bibliographiques \\
\hline I. & Amiens & $\begin{array}{l}\text { Millet, Hélène - Desportes, Pierre (ed.) (1996): Fasti Ecclesiae Gallicanae. } \\
\text { Répertoire prosopographique des évêques, dignitaires et chanoines des diocèses } \\
\text { de France de } 1200 \text { à } 1500 \text {. I. Diocèse d’Amiens. Turnhout, Brepols. }\end{array}$ \\
\hline II. & Rouen & $\begin{array}{l}\text { TabBagh, Vincent (ed.) (1998): Fasti Ecclesiae Gallicanae. Répertoire prosopo- } \\
\text { graphique des évêques, dignitaires et chanoines des diocèses de France de } 1200 \text { à } \\
\text { 1500. II. Diocèse de Rouen. Turnhout, Brepols. }\end{array}$ \\
\hline III. & Reims & $\begin{array}{l}\text { Desportes, Pierre (ed.) (1998): Fasti Ecclesiae Gallicanae. Répertoire prosopo- } \\
\text { graphique des évêques, dignitaires et chanoines des diocèses de France de } 1200 \text { à } \\
\text { 1500. III. Diocèse de Reims. Turnhout, Brepols. }\end{array}$ \\
\hline IV. & Besançon & $\begin{array}{c}\text { Hours, Henri (ed.) (1999): Fasti Ecclesiae Gallicanae. Répertoire prosopogra- } \\
\text { phique des évêques, dignitaires et chanoines des diocèses de France de } 1200 \text { à } \\
\text { 1500. IV. Diocèse de Besançon. Turnhout, Brepols. }\end{array}$ \\
\hline V. & Agen & $\begin{array}{c}\text { Ryckebusch, FABRice (ed.) (2001): Fasti Ecclesiae Gallicanae. Répertoire proso- } \\
\text { pographique des évêques, dignitaires et chanoines des diocèses de France de } 1200 \\
\text { à } 1500 \text {. V. Diocèse d'Agen. Turnhout, Brepols. }\end{array}$ \\
\hline VI. & Rodez & $\begin{array}{c}\text { Desachy, Matthieu (ed.) (2002): Fasti Ecclesiae Gallicanae. Répertoire proso- } \\
\text { pographique des évêques, dignitaires et chanoines des diocèses de France de } 1200 \\
\text { à 1500. VI. Diocèse de Rodez. Turnhout, Brepols. }\end{array}$ \\
\hline
\end{tabular}

12 Une liste des responsables des diocèses est disponible: FEg, Collaborateurs. 


\begin{tabular}{|c|c|c|}
\hline VII. & Angers & $\begin{array}{l}\text { Matz, JeAn-Michel - COMte, François (ed.) (2003): Fasti Ecclesiae Galli- } \\
\text { canae. Répertoire prosopographique des évêques, dignitaires et chanoines des } \\
\text { diocèses de France de } 1200 \text { à } 1500 . \text { VII. Diocèse d'Angers. Turnhout, Brepols. }\end{array}$ \\
\hline VIII. & Mende & $\begin{array}{l}\text { Maurice, Philippe (ed.) (2004): Fasti Ecclesiae Gallicanae. Répertoire prosopo- } \\
\text { graphique des évêques, dignitaires et chanoines des diocèses de France de } 1200 \text { à } \\
\text { 1500. VIII. Diocèse de Mende. Turnhout, Brepols. }\end{array}$ \\
\hline IX. & Sées & $\begin{array}{c}\text { Desportes, PierRe - Fouché, JEAn-PASCAL - Loddé, Françoise -Vallière, } \\
\text { Laurent (ed.) (2005): Fasti Ecclesiae Gallicanae. Répertoire prosopographique } \\
\text { des évêques, dignitaires et chanoines des diocèses de France de } 1200 \text { à } 1500 . \text { IX. } \\
\text { Diocèse de Sées. Turnhout, Brepols. }\end{array}$ \\
\hline $\mathrm{X}$. & Poitiers & $\begin{array}{l}\text { VAllière, LAUrent (ed.) (2008): Fasti Ecclesiae Gallicanae. Répertoire prosopo- } \\
\text { graphique des évêques, dignitaires et chanoines des diocèses de France de } 1200 \text { à } \\
\text { 1500. X. Diocèse de Poitiers. Turnhout, Brepols. }\end{array}$ \\
\hline XI. & Sens & $\begin{array}{l}\text { TabBagh, Vincent (ed.) (2010): Fasti Ecclesiae Gallicanae. Répertoire prosopo- } \\
\text { graphique des évêques, dignitaires et chanoines des diocèses de France de } 1200 \text { à } \\
\text { 1500. XI. Diocèse de Sens. Turnhout, Brepols. }\end{array}$ \\
\hline XII. & Autun & $\begin{array}{c}\text { MADIGNIER, JACQUes (ed.) (2010): Fasti Ecclesiae Gallicanae. Répertoire proso- } \\
\text { pographique des évêques, dignitaires et chanoines des diocèses de France de } 1200 \\
\text { à } 1500 . \text { XII. Diocèse d'Autun. Turnhout, Brepols. }\end{array}$ \\
\hline XIII. & Bordeaux & $\begin{array}{l}\text { LAINÉ, Françoise (ed.) (2012): Fasti Ecclesiae Gallicanae. Répertoire prosopo- } \\
\text { graphique des évêques, dignitaires et chanoines des diocèses de France de } 1200 \text { à } \\
\text { 1500. XIII. Diocèse de Bordeaux. Turnhout, Brepols. }\end{array}$ \\
\hline XIV. & $\begin{array}{l}\text { Châlons-en- } \\
\text { Champagne }\end{array}$ & $\begin{array}{c}\text { Guilbert, Sylvette (ed.) (2014): Fasti Ecclesiae Gallicanae. Répertoire proso- } \\
\text { pographique des évêques, dignitaires et chanoines des diocèses de France de } 1200 \\
\text { à } 1500 . \text { XIV. Diocèse de Châlons-en-Champagne. Turnhout, Brepols. }\end{array}$ \\
\hline \multicolumn{2}{|c|}{ En préparation } & Éditeur \\
\hline \multicolumn{2}{|c|}{ Clermont-Ferrand } & Henri Hours \\
\hline \multicolumn{2}{|c|}{ Toul } & Pierre Pégeot \\
\hline \multicolumn{2}{|c|}{ Le Mans } & Jean-Michel Matz \\
\hline \multicolumn{2}{|c|}{ Narbonne } & Benoit Brouns \\
\hline
\end{tabular}

L'impact du projet des Fasti est bien complexe. L'évaluation qu'André Vauché a formulé en 1996 s'est justifiée pendant ces presque deux décennies : "nous voulions aussi et surtout permettre à tous les médiévistes, quelle que soit leur spécialité, de tirer la meilleur parti de l'énorme documentation accumulée par les ecclésiastiques médiévaux, tant à la base qu'au sommet, et des nombreuses information des tous ordres qu'elle véhicule. Le tome premier des Fasti Ecclesiae Gallicanae répond pleinement à cette attente $»{ }^{13}$ Les travaux effectués par les collègues portugais, les préparatifs de la candidature «eContentPlus » en 2007-2008, les résultats du Groupement de recherche européen ${ }^{14}$ illustrent bien l'écho international

13 VAUCHEZ 1996. VIII.

14 Cf. note nr. 11. 2007-2008 : «LEGACY The European de cette entreprise exceptionnelle. Le méthode qui y est appliqué - recherches basées sur des répertoires prosopographiques et des notices biographiques - se reflète dans plusieurs études traitant de différents domaines les plus variées de la médiévistique des mélanges offertes en l'honneur d'Hélène Millet, créatrice de toute l'entreprise. ${ }^{15}$

cultural legacy of medieval churchmen ", 2010-2013 : «Aux fondements de la modernité étatique en Europe. L'héritage des clercs médiévaux ». Pour plus de détails voir l'article dans le présent volume: Les clercs mediévaux au service de l'État.

15 Cf. les études du colloque organisé en l'honneur d'Hélène Millet : Barralis - Boudet - Delivré GeNET 2014. 


\section{ABRÉVIATIONS, BIBLIOGRAPHIE}

Albanès, Joseph Hyacinthe - Chevalier, Ulysse (ed.) (1899-1920): Gallia christiana novissima. Histoire des archevêchés, évêques et abbayes de France. I-VII. Montbéliard, Paul Hofmann.

Autrand, Françoise (ed.) (1986): Prosopographie et Genèse de l'État Moderne. Actes de la Table Ronde organisé par le Centre National de la Recherche Scientifique et l'École Normale Supérieure de jeunes filles. Paris, 22-23 Octobre 1984. Paris, Editions du CNRS.

Barralis, Christine - Boudet, Jean-Patrice - DeliVRÉ, Fabrice - Genet, Jean-Philippe (ed.): Les chanoines de la fin du Moyen Age étaient-ils au service de l'État? Les clercs et la genèse de l'État moderne. Actes de la conférence organisée à Bourges en 2011 par SAS et l'Université d'Orléans en l'honneur d'Hélène Millet. Paris, Publications de la Sorbonne - École française de Rome. (Publications de la Sorbonne - Collection del'École française de Rome - 485/10 : Le pouvoir symbolique en Occident (1300-1640) - X.)

C. Tóth, NorberT - LAKatos, BÁlint - Mikó, Gábor (2014): A pozsonyi prépostés a káptalan viszálya (1421-1425). A szentszéki biráskodás Magyarországon - a pozsonyi káptalan szervezete és müködése a XV. század elején. Budapest, Magyar Tudományos Akadémia-Hadtörténeti Intézet és Múzeum - Szegedi Tudományegyetem - Magyar Nemzeti Levéltár Országos Levéltára - Magyar Medievisztikai Kutatócsoport. (Subsidia ad historiam medii aevi Hungariae inquirendam, 3).

De Sainte-Marhte, Denis - Hauréau, Jean-BarthéLÉMY (ed.) (1715-1865): Gallia christiana in provincias ecclesiasticas distributa, qua series et historia archiepiscoporum, episcoporum et abbatum Franciae vicinarumque ditionum ab origine ecclesiarum ad nostra tempora deducitur et probatur ex authenticis instrumentum ad calcem appositis. Paris, Joannes-Baptista Coignard.

Duchesne, Louis (1907-1910): Fastes episcopaux de l'ancienne Gaule. I-II. Paris, Fontemoing.

Feg, Collaborateurs: Fasti Ecclesiae Gallicanae, Les collaborateurs des Fasti http://fasti.univparis1.fr/groupe_membres.php (date de consultation: 12/2/2015).

Feg, Conception: Fasti Ecclesiae Gallicanae, Conception http://fasti.univ-paris1.fr/donnees_ conception.php (date de consultation: 12/2/2015).
Feg, Historique: Fasti Ecclesiae Gallicana, Historiquehttp://fasti.univ-paris1.fr/fasti_tutelles.php (date de consultation: 12/2/2015).

Feg, Organigramme: Fasti Ecclesiae Gallicanae, Organisation du groupe des Fasti http://fasti. univ-parisl.fr/groupe_organigramme.php (date de consultation: 12/2/2015).

Feg, Réunions: Fasti Ecclesiae Gallicanae, Réunions de l'équipe des Fasti http://fasti.univ-paris1. fr/groupe_reunions.php (date de consultation: 12/2/2015)..

Feg, Structure: Fasti Ecclesiae Gallicanae, Structure d'un volume http://fasti.univ-paris1.fr/ collection_structure.php (date de consultation: 12/2/2015).

Fedeles, Tamás - Sarbak, Gábor - Sümegi, JózseF (ed.) (2009): A középkorévszázadai (1009-1543). A pécsi egyházmegye története I. Pécs, Fény Kft.

KIss, Gergely (2014): Les chanoines de la fin du Moyen Age étaient-ils au service de l'État ? La Hongrie. Bilan et perspectives. In : BARRALIS - BOUDET Delivré - Genet 2014. 127-135.

LAmop : Laboratoire de Médiévistique Occidentale de Paris http://lamop.univ-paris1.fr/ (date de consultation: 12/2/2015).

Millet, HÉlÈne (ed.) (1985): Informatique et Prosopographie. Actes de la Table Ronde du CNRS, Paris, 25-26 Octobre 1984. Paris, Editions de CNRS.

Millet, HÉLÈne (1996): Présentation de la collection. In: MiLLET - DESPORTES. IX-X.

Millet, Hélène - Desportes, Pierre (ed.) (1996): Fasti Ecclesiae Gallicanae. Répertoire prosopographique des évêques, dignitaires et chanoines des diocèses de France de 1200 à 1500. I. Diocèse d'Amiens. Turnhout, Brepols.

Millet, HÉlÈne - RauX, Stéphan (2007): De la publication de livres à la mise en ligne d'une base de données. Les premiers enseignements du puzzle des Fasti Ecclesia Gallicanae. In: Carreiras Eclesiásticas no Ocidente Cristão (séculos XII-XIV). Ecclesiastical Careers in Western Christianity $\left(12^{\text {th }}-14^{\text {th }} c\right.$. $)$. Lisboa, Centro de Estudos de História Religiosa - Universidade Católica Portuguesa. 317-330.

VAuchez, ANdré (1996): Avant-propos. In: Millet, Hélène - Desportes, Pierre (ed.): Fasti Ecclesiae Gallicanae. Répertoire prosopographique des évêques, dignitaires et chanoines des diocèses de France de 1200 à 1500. I. Diocèse d'Amiens. Turnhout, Brepols. VII-VIII. 